\title{
VISUAL PERCEPTION IN JAPANESE ROCK GARDEN DESIGN
}

\begin{abstract}
We present an investigation into the relation between design principles in Japanese gardens, and their associated perceptual effects. This leads to the realization that a set of design principles described in a Japanese gardening text by Shingen (1466), shows many parallels to the visual effects of perceptual grouping, studied by the Gestalt school of psychology. Guidelines for composition of rock clusters closely relate to perception of visual figure. Garden design elements are arranged into patterns that simplify figure-ground segmentation, while seemingly balancing the visual salience of subparts and the global arrangement. Visual 'ground' is analyzed via medial axis transformation (MAT), often associated with shape perception in humans. MAT analysis reveals implicit structure in the visual ground of a quintessential rock garden design. The MAT structure enables formal comparison of structure of figure and ground. They share some aesthetic qualities, with interesting differences. Both contain naturalistic asymmetric, self-similar, branching structures. While the branching pattern of the ground converges towards the viewer, that of the figure converges in the opposite direction.
\end{abstract}

KEY WORDS: figure-ground, multi-scale, segmentation, tree structure, visual grouping

"There is ... a basic issue that is only very seldomly discussed: What do we mean by "visual experience"? Very often the problem of vision is oversimplified, and to "see" a scene is identified with the task of computing a verbal description of it. This problem is difficult enough, but it is important to recognize that there is much more in visual perception than assigning verbal labels to "objects". '

J.L. Marroquin (1976)

\section{INTRODUCTION}

Japanese gardens are well known for their sophisticated, minimal design and for the calm yet profound atmosphere which they engender. After an opportunity to witness garden designers at work, the authors realized that successful garden composition partly results from the application of intentional, complex design principles, with anticipated visual effects. We therefore asked how the 
visual elements of a garden, its rocks, moss and shrubs, and the compositions that gardeners create, are interpreted by the brain. How do gardens achieve particular expressive and artistic effects?

In this paper we consider possible implications of intentional garden design effects, by interpreting how design principles affect two of the fundamental processes associated with human visual perception, namely segmentation of the visual scene into meaningful parts, and perceptual grouping of parts into meaningful structural wholes. We suggest that principles of visual psychology shed new light on the aesthetic issues related to Japanese garden design.

\section{INTENTIONAL DESIGN EFFECTS IN JAPANESE GARDENS}

Japanese garden designers, today, would be familiar with the following list of the key techniques used in garden design:

- Rocks form the backbone of the garden composition.

- Triangular rocks and rock clusters are preferred. As compositional units, or so-called triads, they express earth, man and the divine through horizontal, diagonal and vertical lines, respectively.

- Odd-numbered groupings of rocks are preferred; the total number of clusters should also be an odd number.

- The largest rock in each cluster is set first. Smaller rocks must then be placed such that they are in 'good agreement' with the main rock of each cluster.

- The composition can be rounded off through various techniques of adding rocks. For example, 'base stones' are typically placed at the foot of the dominant rock in a cluster, to extend its base and make it look more triangular; Sute ishi, literally meaning thrown away stones, are low, inconspicuous stones that appear as if scattered in a random manner. These techniques are used to make a composition look more natural.

- Arranging rocks like the scales on a fish (also called the folding screen technique), creates the impression of a vast, deep landscape with mountains.

- Suggested landscape features, like streams, should never be straight, but curved in a manner suggestive of an endlessly winding structure.

- Asymmetry of all aspects of the design should be considered. 
- Design elements, such as rocks, moss and gravel, should have uniform textures, without bright colouration or excessively striking textural patterns.

Origins of the above list of design principles can be traced back to two gardening manuals, the earliest being a medieval text known as the Sakuteiki, translated by Shimoyama (1976). The other, an illustrated text (Shingen, 1466), dates from the Muromachi (1333-1573) period. The latter text contains both verbal and diagrammatic descriptions of dry landscape gardens in particular. The previous list of design principles is actually a summary of the main tenets of the text by Shingen.

\section{THE VISUAL PSYCHOLOGY OF PERCEPTUAL GROUPING}

The Gestalt psychologists (Koffka, 1935; Wertheimer, 1938) stressed the importance of the process whereby the human brain groups together various visual cues into meaningful perceptual wholes. Visual grouping can be interpreted as segmentation, the division, by the visual system, of a scene into possibly meaningful parts, as an early step in the analysis of a visual scene. Koenderink et al. (1992) analyzed the segmentation process further into the active description of a scene, in terms of the surface regions and bounding contours of objects. At this level, local contour elements and surface texture elements are grouped into outlines and regions of segments, respectively. In a more complex stage of grouping, the segmented image is arranged into figure and ground - the next step in the analysis of a visual scene. The process of visual segmentation, central and fundamental to the understanding of human vision, is not yet completely understood. The Gestalt school provided a set of intuitive guidelines for predicting the outcome of perceptual grouping, that is, the formation of meaningful arrangements of visual elements. The most fundamental independent guidelines, often called Gestalt laws, are still relevant in perception research today (Palmer, 1999), and is summarized by the following (see Figure 1A1-A5 for simple examples):

- Proximity: More closely spaced cues are seen as belonging together

- Similarity: Elements that look more similar are grouped together

- Smoothness: Elements group together if their spatial alignment follows a smooth path 

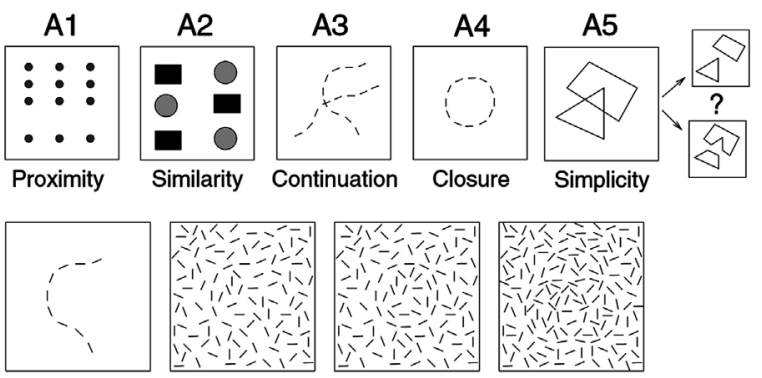

B3

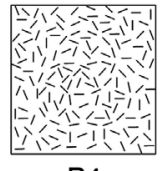

$1: 1$

$1: 5$

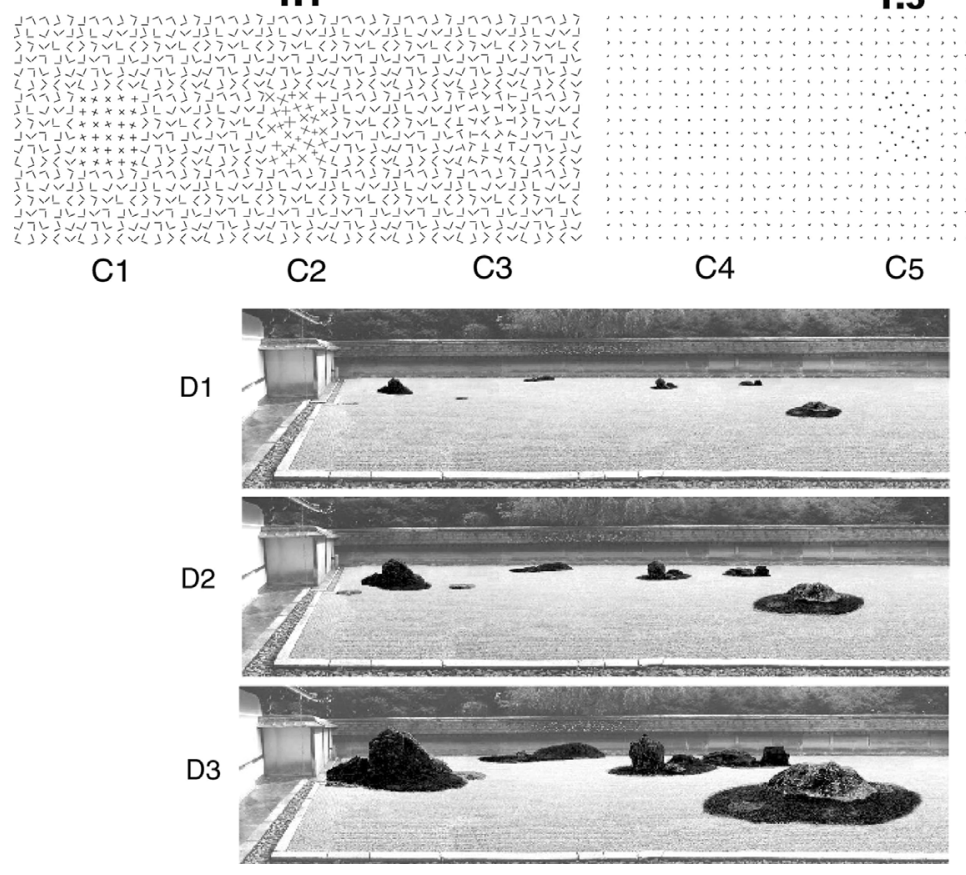

Figure 1. Gestalt laws are intuitive (A1-A5). Visual grouping is non-linear. Adding more visual cues lead to complex effects in the final percept (B1-B4). At a cue: size ratio of $1: 1$, target $\mathrm{C} 1$ is more salient than $\mathrm{C} 2$ and $\mathrm{C} 3$. At a ratio of $1: 5$ the same target, now $\mathrm{C} 4$, no longer segments from the background, while $\mathrm{C} 5$ pops out. Tessellation of the texture grid, i.e. its global structure, is now clearly visible. The Ryoanji design (D1) was recreated at a cue: space ratio of 1:5, at the normal ratio (D2) of 1:2 and 1:1 (D3).

- Enclosedness: Objects group together if they are arranged on a closed path

- Simplicity law: The objects actually perceived correspond to the ecologically simplest configuration of parts. 
The above does not imply that distinct features independently affect the grouping of visual elements into boundary contours and surface regions. Rather, the superposition of features with different gestalt qualities is not linearly additive, but complex. Gestalt formation at one scale affects gestalt formation on another. The smooth curve in Figure 1B1 is not salient among distractors (Figure 1B2), while a closed contour is effortlessly perceived (Figure 1B3). Addition of more closed contours reduces this pop-out effect (Figure 1B4).

Similarly, the proximity factor has complex implications for grouping. For example, the formation of boundary contours, and hence image segmentation, is affected by the ratio of visual cue size to the spatial distances between them. Zucker and Davis (1988) found a salient change in contour perception at a cue size: empty space ratio of about 1:5. More sparsely sampled contours fail to generate various classical illusions associated with the reconstruction of incomplete boundary contours. Cues on such sparse contours fail to be integrated into a whole figure (Kovács, 2000).

Caelli and Julesz (1978) proposed that local visual cues are organized into texture elements, or so-called textons, which represent local geometrical characteristics of the given visual cues. They suggested that boundary contours, formed during image segmentation, are based on gradients in texton distribution. Their theory explains why the target in Figure $1 \mathrm{Cl}$ clearly segments from the surround, while Figure 1C3 does not. In the first case, local geometry of line crossing textons is sufficiently different from the blind angular junctions of the ' $\mathrm{L}$ ' shaped textons, but in the latter, both ' $T$ ' and ' $L$ ' textons support the same local geometry and do not segment.

Van Tonder and Ejima (2000) have argued that tessellation, and hence the ratio of cue size of textons to the relative spacing between them, strongly influences visual grouping. First, the local geometry of the empty spaces between textons also plays a role in perceptual grouping. Figure 1C2, with the same local texton geometry as Figure $1 \mathrm{C} 1$, is not as visually salient, since the local geometry of empty spaces in Figure $1 \mathrm{C} 1$ is regular, forming a strong gradient with the surrounding irregular texture. In comparison, local geometry of empty spaces and their surrounding textons (Figure 1C2) are all irregular, and hence the lower visual salience 
of this targe against its background. Thus, local geometry, of both the textons and empty spaces between them, determines how proximity contributes to perceptual grouping.

Secondly, the effect of the relative cue size: empty space ratio is easily demonstrated by comparing Figures $1 \mathrm{C} 1-\mathrm{C} 3$ (with a ratio of about 1:1) with Figures 1C4-C6 (ratio of about 1:5). The latter figures present the same targets as in the first three examples, with textons placed on the same tessellation grid, but here each individual texton is scaled to one-fifth of its original size. The left most and right most targets (Figures 1C4 and C6) are now both indistinguishable from the background, irrespective of their local texton geometry. The geometry of empty space dominates perceptual grouping, and the regularity of the tessellation prevents segmentation. Target Figure 1C5 is now the most salient, as its local geometry differs the most from the background.

At specific intermediate cue size: empty space ratios one may find that local geometry of both the texture elements and the empty spaces between them contribute equally to perceptual grouping of the whole design. It is interesting to note that in a minimalist rock garden like Ryoanji the relative cue size: empty space ratio for rock clusters and the spaces between them is roughly 1:2, between the ratios of 1:1 and 1:5 as shown above. The implications are dealt with the next section.

\section{DESIGN EFFECTS RELATED TO VISUAL FIGURE}

\subsection{Texture effects}

Japanese gardeners use comparatively homogeneously textured materials (Figure 2A). Saturated colours and high contrast texture markings are avoided, possible since low contrast objects tend not to dominate visual attention. The textures used are natural, and therefore visually more complex than a mere blank slate. Uniform surface regions with even textures simplify the creation of boundary contours. The process of visual segmentation is thus simplified through the reduction of the number of sub-segments within the interior of each object. The use of coarse, light-and-dark mottled gravel as background for most of the garden somewhat reduces the 


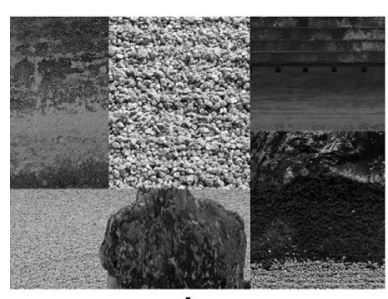

A

C1
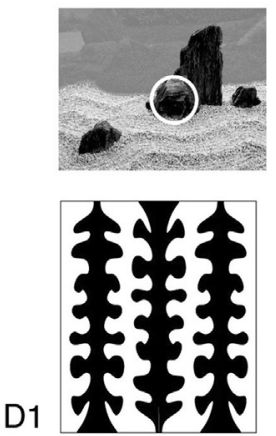

$\mathrm{D} 2$

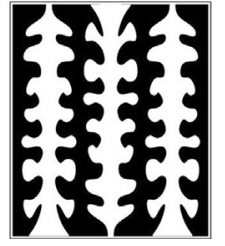

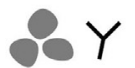

B1 - odd B2 - even

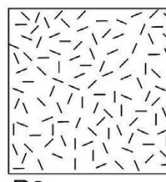

B3
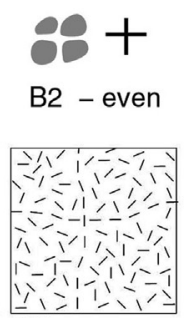

B4

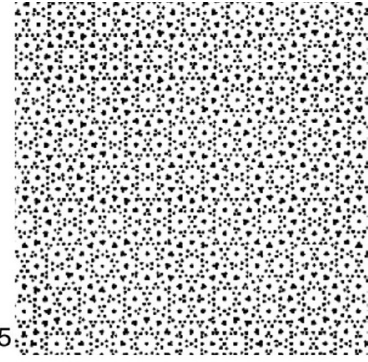

C4

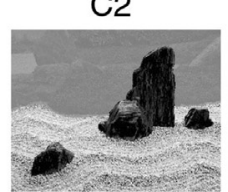

C3
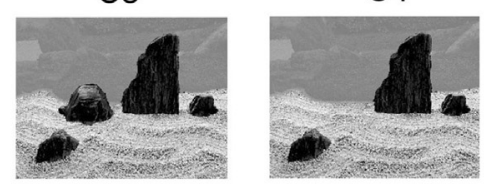

D3

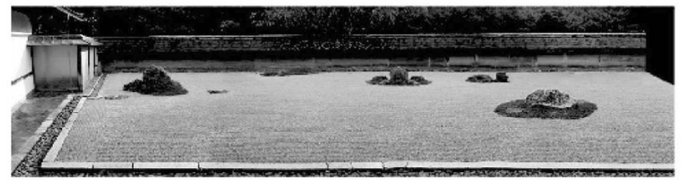

D4

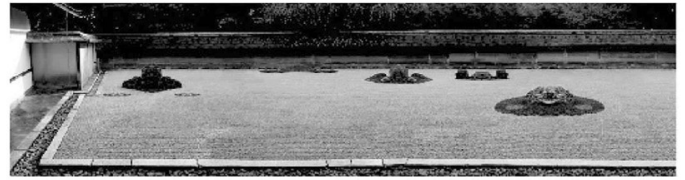

D5

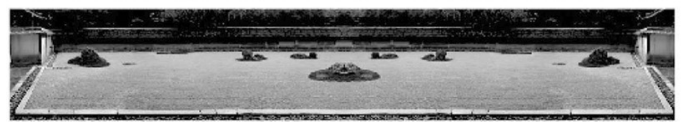

Figure 2. (A) Examples of textures preferred by Japanese gardeners. (B1) Oddnumbered groupings of stones usually result in odd-numbered visual junctions, which disappear (B3) among distractors, whereas even arrangements (B2) lead to even junctions that are more salient (B4). Patterns with many even junctions (B5) result in competing figures. A base stone (white circle) (C1) strengthens visual grouping within the cluster $(\mathrm{C} 2)$. Deliberate alignment (C3) creates an unnatural looking closed figure. Without a base stone the composition visually appears as two separate groups (C4). (D1, D2) Images after Bahnsen (1928) demonstrate how bilateral symmetry dictates to figure-ground perception. (D3) Inducing bilateral symmetry in one rock cluster causes it to 'pop out' in the Ryoanji design. (D4) Local bilateral symmetry weakens grouping between clusters. (D5) More subtle effects of similarity and proximity are dominated by artificially induced global bilateral symmetry.

unavoidable contrast created between rocks and visual ground. Both the rocks and moss have a similarly mottled appearance. The 
contrast between figure and ground would be much greater if fine white sand were used instead.

\subsection{Spacing between rocks}

The garden manual by Shingen (1466) expresses the maximum size of rocks as a ratio of the size of the garden as a whole. The extent to which rocks visually fill a garden will affect the perception of spatial dimensions of the entire garden. Adaptations to spacing between rocks can thus be used to create various moods, such as emptiness or liveliness.

Just as with the previously shown texton textures (Figure 1C), the degree to which stones group depends on the size ratio of the rock arrangement as a whole compared to that of individual rocks. Figure 1D2 shows the rocks and empty spaces in Ryoanji. The ratio of rock cluster size and the distance to its nearest neighbour is 1:2 on average. Downscaling of rock clusters (i.e. decreasing the ratio to 1:5, Figure 1D1) exposes the tessellation pattern of the global composition, but impairs the clarity of visual relationships between individual objects in rock clusters. With the rocks scaled to appear much larger (1:1, Figure 1D3), clusters form a closely knit unit in which subparts of clusters, rather than clusters themselves are visually discernable, and the tessellation scheme of the overall pattern becomes obscured.

\subsection{Contour junctions}

Gardeners carefully consider the visual appearance of spatial junctions (boundary contours) between rocks, as well as surface textures of rocks themselves (surface regions). Visual junctions are created in such a way that an odd number of contours meet (e.g. Figure 2B1), whereas even numbered junctions are avoided (Figure 2B2). According to the Gestalt law of good continuation, an even junction will appear more perceptually salient than an odd junction if seen against a background of random texture elements, such as shown in Figures $2 \mathrm{~B} 3$ and 2B4. Adjusting the formation of odd and even junctions gives gardeners control over the salience of contours, and hence, the process of visual segmentation. Where even junctions abound, such as the pattern in Figure 2B5 (after Marroquin) segmentation is ambiguous: instead of shifting across the image in a structured manner, visual 
attention drifts between different competing figures without revealing any additional global structure.

Traditionallly, the technique of arranging the rocks to overlap alternately, like scales on a fish, was considered evocative of space and depth. Such overlap gives the impression of a series of folds jutting out behind one another along a deep mountain valley (Figure 3A4). The role of occlusion junctions as vivid monocular depth clues is known in visual psychophysics. Alternating overlap of rocks ensures the creation of odd (or trilateral) junctions.

\subsection{Extending the spatial range of grouping}

Placement of smaller rocks facilitates subtle adjustments of grouping within and between clusters. Sute ishi, literally meaning 'thrown away stones', are low rocks (see the left most rock cluster in
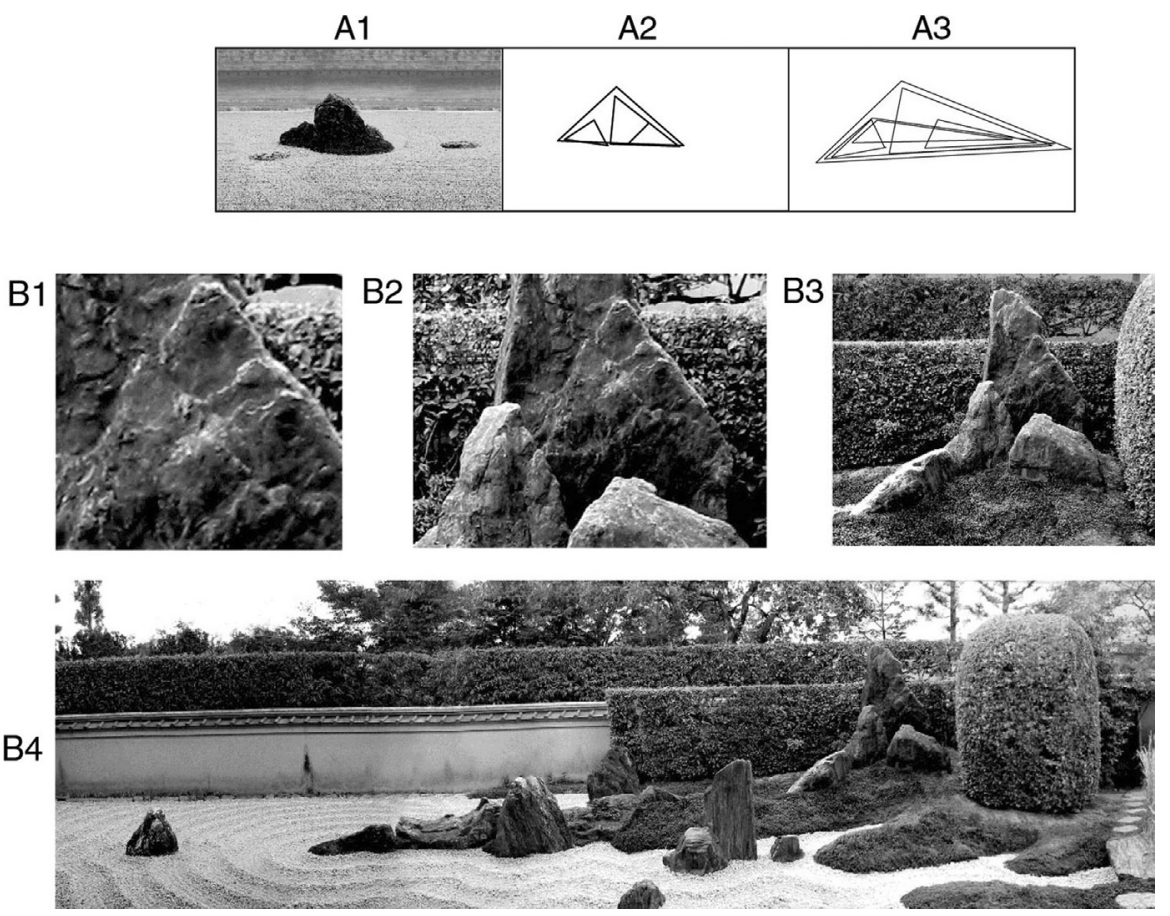

Figure 3. (A1) Sute ishi, or 'thrown away stones' (A1) increase the number of triangular groupings in the original rock cluster (A2) to extend more widely into the rest of the garden (A3). An enlarged view of rock surface texture (B1) and successively more global views (B2-B4), reveal a recurrence of triangular arrangements in this garden (Dokuzatei, Kyoto). 
Figure 1D2, or Figure 3B1), inconspicuously placed within or around a rock cluster. Grouping with the rest of the global garden composition is thus reinforced, since the cluster extends over a wider range. Without these stones, clusters would appear more as closed figures. The Gestalt law of closure predicts that such arrangements would stand alone rather than visually grouping with other elements in the overall design.

Figure $2 \mathrm{C} 1$ demonstrates another technique to alter grouping: the use of stones placed at the foot of the dominant rock in a cluster, to extend its base, thereby reinforcing the grouping of stones within the cluster (Figure 2C2). Though four stones are used here (an even number), visually they constitute a single group with a triangular composition and odd numbered junctions. In Figure 2C3 the composition is arranged into a semi-circle, breaking self-similarity, with a less natural looking result. Removal of the base rock (Figure 2C4) weakens the grouping between the left and right side of the composition so that it distinctly appears as two separate clusters, which is almost certainly not what the designer of this rock arrangement intended.

\subsection{Asymmetry}

Japanese art emphasizes bilateral asymmetry in design. Bahnsen (1928) and Koffka (1935) demonstrated the strong effect of bilateral symmetry on figure-ground segmentation. Instead of perceiving the textures in Figure 2D1 and 2D2 as patterns of equal black and white bands, humans consistently see the most bilaterally symmetrical forms as figures lying in front of a uniform background. Luminance contrast can be reversed without affecting the bias of bilateral symmetry on figure-ground segmentation, in this example. Bilateral symmetry is independent of proximity, similarity and smoothness - all local qualities of visual cues. A shape can also be spatially closed and very simple, yet these conditions do not guarantee bilateral symmetry of the shape in question.

From the example in Figures 2D1 and 2D2 after Bahnsen (1928), it is clear that symmetrical objects dominate figure ground segmentation and therefore could jeopardize visual perception of subtle aspects in gardens. If one rock cluster is bilaterally symmetrical, it tends to visually stand out from other clusters (Figure 2D3). Grouping between bilaterally symmetrical rock clusters is compromised because each cluster now 'pops out' as a 
separate figure, vying for attention (Figure 2D4). A garden with global bilateral symmetry imposed (Figure 2D5) looks clearly different from the original, asymmetrical design where the various visual interactions between rock clusters tend to find their own, more natural, equilibrium.

\subsection{Self-similarity and multi-scale grouping}

Natural patterns are often self-similar, the outcome of repeated application of the same organizing principle at multiple spatial scales. The examples of rock compositions shown previously display self-similarity in that a triangular theme is repeated at several scales. In Figures $3 \mathrm{~A} 1$, each rock is itself roughly triangular, as outlines of rock shapes show (Figure 3A2). Sute ishi, the low stones in Figure 3A1, echo the triangular appearance of the central composition by allowing the formation of many possible triangular groupings at different spatial scales within the rock cluster (Figure 3A3), thereby lending a degree of self-similarity to the triangular design. In the example with a base extending stone (Figure 2C2), the three most proximate rocks to the right form a triangle, while the fourth, left most stone extends the composition into a larger triangle. The central rock, or base stone, significantly increases the number of possible triangles that can result from grouping between nearest neighbouring rocks.

The technique thus reinforces grouping between and within rock clusters by tuning of self-similarity and proximity between visual cues. Note that the orientation of asymmetry (towards the left or the right) of different triangular groupings alternates both within the same spatial scale and between different spatial scales. Duplication, causing visual bias towards any part of the design, is thus avoided. In both examples the additional stones increase the degree of bilateral asymmetry in the relevant rock clusters. Hence the technique further reduces the visual salience of rock clusters by breaking their bilateral symmetry.

Figure 3B4 shows the Dokuzatei garden at Diatokuji in Kyoto. Self-similarity of the rock composition is evident over many spatial scales, and extends to the level of surface texture. In Figure 3B1 the textural markings of a rock, shown close-up, echo the triangular shape of rocks. Figures 3B2-B4 show the triangular arrangements at increasingly larger scales. The Gestalt law of similarity predicts that the repetition of these shapes and their visual 
junctions would facilitate grouping. Although similar shapes reappear over multiple scales, the shapes themselves are irregular, preserving the asymmetrical, natural appearance of the overall design. Repetition of the pattern of overlap between rocks is also visible at multiple scales. Note that the junctions created by overlap are all odd, avoiding rivalrous segmentation at any single junction. The arrangement of junctions in the global figure resembles an inverted tree structure with dichotomous branching points. This type of structure often occurs in natural landscape formations, such as mountains and valleys. Proponents of more organic, humanistic architecture have argued convincingly in favour of multi-scale architectural design (Alexander, 1979; Salingaros, 2000).

\section{ANALYSIS OF VISUAL GROUND}

Various ideas on the organization of visual figure was presented in the previous sections. Here, we outline our approach to analyze the structure of visual ground, and relate visual ground with visual grouping in more general terms.

\subsection{Medial axes and human perception}

Various recent discoveries point toward a link between human visual perception and medial axis representation of shape. To understand the medial axis transform intuitively, imagine a fire burning through a field of grass (Blum, 1973). For example, fires started simultaneously at two different points would eventually meet and stop burning along a line, equidistant from both starting points (Figure 4A1). This line is the medial axis between the two points. When the fire is started along a closed contour in the field of grass, such as the tri-lobed shape in Figure 4A2, it will propagate inwards until stopping at loci of axial symmetry. In the example, such loci are visible as dark lines in Figure 4A2.

Kovacs and Julesz (1994) discovered that human luminance contrast sensitivity is enhanced along loci that correspond exactly to the medial axis of the given stimulus figure. For a triangular shape, shown as a white outline in Figure 4A3, human contrast sensitivity increases along the corresponding medial axes shown in black. Horizontal and vertical cross sections of the computed medial axis are shown. Kovács et al. (1998) found that human contrast sensitivity measurements are closely matched by computational medial 


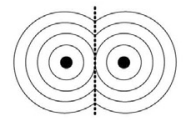

A1

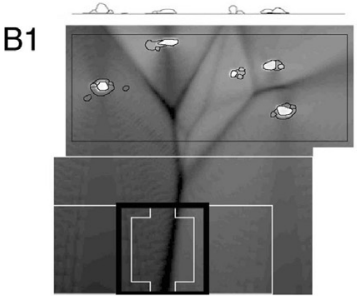

B3

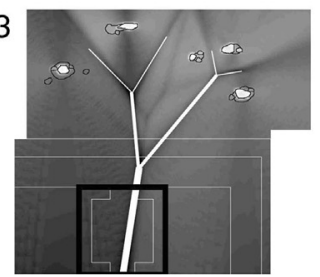

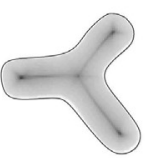

$\mathrm{A} 2$
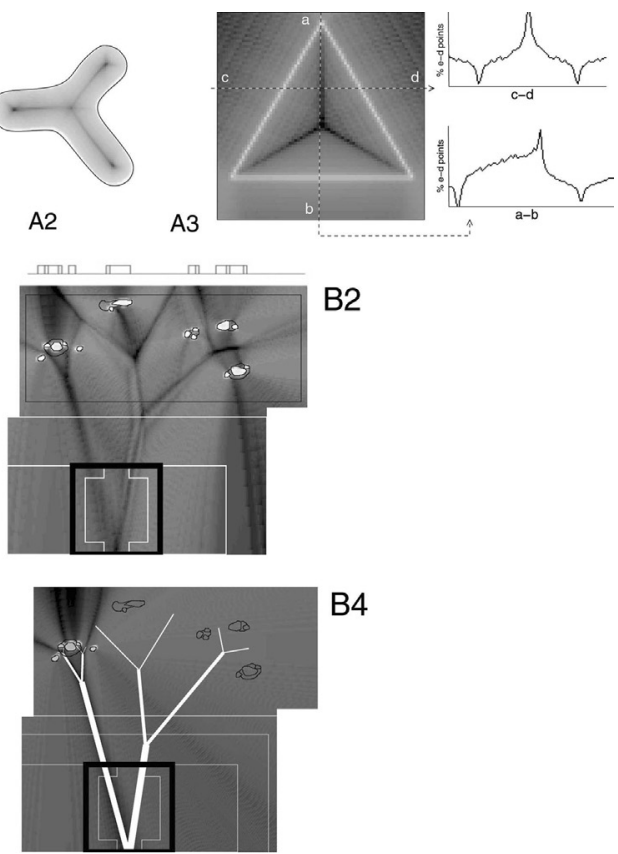

B2

B4

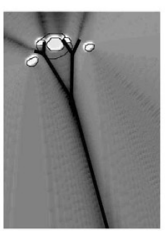

C1

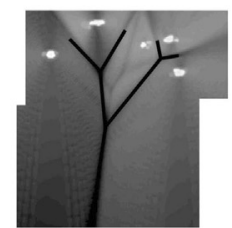

C2

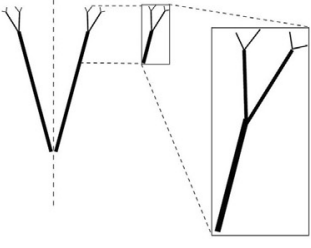

C3

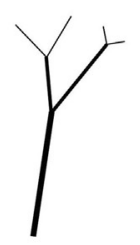

C4

Figure 4. (A1) The medial axis between two points is the locus of equidistant points between them. (A2) A two-dimensional shape (dark outline) and its medial axis transform (internal shading) computed with our model. (A3) Contrast sensitivity enhancement data of Kovács et al. (1998) of a triangle, with vertical and horizontal cross sections to show data in detail, were replicated with the model by Van Tonder and Ejima (2003). (B1) Medial axis transformation of Ryoanji. The layout is after Oyama (1995). The outlines of rocks appear within the rectangle of gravel. The verandah and main hall are also outlined. The central room appears as a bold black outlined square. Rock contour values are scaled to relative rock heights. (B2) The simulation, with rock contour values all set equal. (B3, B4) The medial axes for the original simulation and perturbed simulations are highlighted in white, for clarity. (C1) An enlarged view of the medial axis tree of the left most rock cluster. (C2) The global medial axis tree. (C3) Horizontal reflection and enlargement of a section of the local tree reveals a close similarity with the global structure (C4). 
axis transform data, indicating that the correlation is not just vaguely similar. Humans do not consciously perceive medial axes in a visual scene, but the findings of Kovacs et al. demonstrate that, unconsciously, our vision is sensitized along these axes. Medial axes constitute compact description vectors of shape and the visual brain may use them for this purpose.

\subsection{Analysis of the structure of visual ground in a Japanese rock garden}

Medial axis transformation is not only useful for compact description of shape regions, but also reveals structure in empty spaces between figures. It could therefore be an appropriate method to analyze minimalist designs often encountered in Japanese dry landscape gardens. A summary of our previous work on structural analysis of the Ryoanji dry rock garden (Van Tonder et al. 2002) is presented here.

Ryoanji hardly requires an introduction as it is well known among designers as well as the lay public. With five clusters of rocks on a rectangular bed of raked gravel (Figure 1D8), visitors to the garden are intrigued by the sophisticated simplicity of the nearly empty composition.

Compared to more typical garden designs, the visual ground in Ryoanji is strongly emphasized. It covers the full extent of the garden. Looking for structure in the empty regions between rock clusters therefore seemed like a reasonable way to proceed in the analysis of the garden's global shape. Medial axis transformation revealed a well defined global pattern (Figure 4B1) with several unusual qualities.

The medial axes constitute a global structure that converges toward the main viewing area, forming a very simple tree shape with three hierarchical branching levels (with a main trunk, two secondary branches and four tertiary branches). The branching point closest to the building, where the tree bifurcates into the first two branches, is a location of maximal global Shannon information (Leyton, 1987). The structure of the entire garden design can therefore be described most compactly at this point. The transformation reveals a self-similar dichotomously branched structure, with a naturalistic appearance reminiscent of both organic (from the myriad of phytomorphic and zoomorphic examples) and inorganic (geological and meteorological formations) patterns of nature. The 
shape of the tree is asymmetrical. The same computational model (Van Tonder and Ejima, 2003) for medial axis transformation was used throughout with a fixed set of parameters, adjusted to fit the human perceptual data described in Kovács et al. (1998).

Medial axes do not converge towards the viewing area upon deletion or addition of clusters, or following random placement of the five rock clusters. After such alterations, medial axes of the garden lack a consistent branching rule and in most cases do not even approximately resemble a tree structure (supplementary material, Van Tonder et al. 2002). The structure defined by the shape of the ground in the original layout of Ryoanji is nonaccidental.

The input stimulus was perturbed to test the robustness of the computational model used. Whereas boundary contours of rocks where scaled to their corresponding relative heights in the initial computation, we set contours of both small and large rocks to equal values. The resulting medial axis transform (Figure 4B2) shows that the global tree structure is still largely the same, except for the addition of small, noisy branches. The main medial axes are highlighted in white (Figures 4B3, B4) for clarity.

Note that the left most rock cluster in the perturbed version produces a branch that meets the main trunk of the original tree structure (Figure 4B4) in the main viewing area of the garden. Closer inspection reveals that this local tree (Figure 4C1) shares various qualities with the global (Figure 4C2) tree structure. For illustrative purposes we horizontally reflected and enlarged a section of the local tree (Figure 4C3). Both local and global (Figure 4C4) structures are self-similar dichotomous branching patterns of the same hierarchical order, and both converge towards the same viewing area.

\section{DISCUSSION AND CONCLUSION}

A Muromachi-era gardening manual (Shingen, 1466) contains drawings of rock configurations, and verbal descriptions of their associated visual effects. Our presentation here resulted from the realization that these guidelines are analogous the Gestalt laws of visual perception. We applied Gestalt principles of perceptual grouping to gain new insight into how karesansui design deals with aesthetic qualities such as asymmetry, tranquility, simplicity and naturalness (Nitschke, 1993). We hypothesize that gardeners 
manipulate the process of visual segmentation into figure and ground by controlled use of various design elements. Specifically, they engender naturalness and avoid excessive local pop-out of visual elements. Likewise, symmetry, even-numbered junctions and excessive heterogeneity with respect to scale transformations lead to unnaturally complicated scenes which disrupt visual tranquility, and goes against the creation of naturalistic design.

Our investigation provides a new interpretation of structure in Japanese garden design. Structure is embedded in hierarchies of trilateral junctions arranged into multi-scale, dichotomously branching patterns in both visual figure and ground, with the main difference being that, while figure converges away from the viewer, the structure of ground converges in the opposite direction. The human sense of aesthetic is closely linked to visual perception. Since the latter comprises an understanding of visual structure, our work therefore suggests a new link between structure and aesthetic understanding of this type of garden design.

Our analysis of structure in Ryoanji shows interesting, although indirect, similarities to the approach developed by Leyton (1992) to interpret works of art. Guided by his theorem on the duality of contour and symmetry, Leyton uses the insight that contours and medial axes coincide at curvature maxima, and use such points and their associated medial axes as the origins and time lines from which the art works can be analyzed as a spatio-temporal evolution of structure.

Our work can be fruitfully compared to that of Taylor et al. (1999), who discovered and measured the degree of statistical selfsimilarity in the structure of visual figure in the drip paintings of Jackson Pollock. In our work, medial axis transformation was applied to uncover the implicit structure of the visual ground. The resultant pattern exhibit various non-accidental qualities, such as self-similarity.

We suggest that the analysis of the Ryoanji garden presented here provides a novel extension to Arnheim's earlier ideas in terms of order and complexity in the garden (Arnheim, 1966). He pointed out that classical French gardens typically employ axial symmetry in the form of a focal point that centers on a radially symmetrical section of the garden and a central axis or lane, leading to the focal centre, whereas Japanese rock gardens do not. Yet application of the medial axis transformation to the Ryoanji design showed that the empty space in much of the garden is highly ordered. The layout 
of this garden forms a global hierarchy with a central point of structural confluence from which a single axis extends. Japanese rock gardens are hence closely related to formal classical French garden design, on an abstract level.

Arnheim correctly asserts that the different rock clusters in Ryoanji are coordinated with each other, rather than forming a central, holistic figural shape. However, our analysis of visual ground reveals that hierarchical structure resides not only within each cluster, but extends from clusters across the entire garden. Even if perception of medial axes remains at a subconscious level, the branching structure gives a clear sense of the holistic shape of the design, which moreover reflects the inverted branching structures suggested by the textural features and triangular compositions of each rock cluster. Medial axis transformation thus enables formal comparison of order and complexity in rock clusters and the entire design.

The concept of $M A$ is an important aspect of Japanese design in more than one way (Nitschke, 1966), yet the best understanding of $M A$ remains at an intuitive rather than a rational level. While we do not argue against this approach, as visual psychologists we are curious to know why the empty space or intervals between objects should have an impact on our visual perception. The subtlety of effects hints at perceptual mechanisms working at a subconscious level and in this investigation we may have established a meaningful link between perceptual mechanisms in vision and the $M A$ in Japanese dry landscape design.

Designers have to simultaneously deal with objects and their surrounding empty spaces. Good designers probably do this effectively, already. However, we would like to suggest a reversed approach of conceptualization, where the structure of empty space is designed first. The resemblance between the tree structure in Ryoanji and natural materials used in other art forms, such as ikebana, suggests direct applicability of techniques from one discipline on another. For example, the idealized branching patterns created in ikebana can be used as the scaffold of empty space for a potential garden, after which the actual placement of rock clusters can be conceived. This may sound counter intuitive at first, but the implied role of empty space in our perception of holistic composition makes it a potential tool for the creation of more harmonized designs. The well defined structure of local symmetry axes could 
assist designers to better understand processes they may already use semi-intuitively.

\section{ACKNOWLEDGEMENTS}

The authors gratefully acknowledge the support of the Japan Society for Promotion of Science and the Japanese Ministry of Education (GJVT), and the National Institute for Communications Technology (MJL). We furthermore would like to express our gratitude to Professor Yoshimichi Ejima, under whose auspices this research was conducted.

\section{REFERENCES}

Alexander, C.: 1979, The Timeless Way of Building, Oxford University Press. Arnheim, R.: 1966, Toward a Psychology of Art, Berkeley: University of California Press.

Bahnsen, P.: 1928. 'Eine Unterschung uber Symmetrie und Asymmetrie bei visuellen Wahrnehmungen', Zeitschrift fur Psychologie 108, 129-154.

Blum, H.: 1973. 'Biological Shape and Visual Science (Part I)', Journal of Theoretical Biology 38, 205-287.

Caelli, T. and B. Julesz: 1978. 'On Perceptual Analyzers Underlying Visual Texture Discrimination: Part I', Biological Cybernetics 28(3), 167-175.

Koenderink, J.J. A.J. Van Doorn and A.M. Kappers: 1992. 'Surface perception in pictures', Perception \& Psychophysics 52(5), 487-496.

Koffka, K.: 1935, Principles of Gestalt Psychology, New York: Harcourt, Brace \& Co.

Kovács, I.: 2000. 'Human development of perceptual organization.', Vision Res. Special Issue on Attention 40(10-12), 1301-1310.

Kovács, I., A. Feher and B. Julesz: 1998. 'Medial-point Description of Shape: A Representation for Action Coding and its Psychophysical Correlates', Vision Research 38(15/16), 2323-2333.

Kovács, I. and B. Julesz: 1994. 'Perceptual Sensitivity Maps within Globally Defined Visual Shapes.', Nature 370, 644-646.

Leyton, M.: 1987. 'Symmetry-Curvature Duality', Computer Vision, Graphics, and Image Processing 38, 327-341.

Leyton, M.: 1992, Symmetry, Causality, Mind, MIT Press: Cambridge, MA, USA. Marroquin, J.L.: 1976, Human Visual Perception of Structure. Master's thesis, MIT. Nitschke, G.: 1966. 'MA: The Japanese Sense of 'Place', Architectural Design 36, $116-156$.

Nitschke, G.: 1993, Japanese Gardens, Koln: Benedikt Taschen Verlag GmbH.

Oyama, H.: 1995, Ryoanji Sekitei: Nanatsu no Nazo wo toku (Ryoanji Rock Garden: Resolving Seven Mysteries). Tokyo: Kodansha. 
Palmer, S. E.: 1999, Vision Science: from Photons to Phenomenology, Bradford Books/MIT Press: Cambridge.

Salingaros, N.: 2000. 'Hierarchical Cooperation in Architecture, and the Mathematical Necessity for Ornament', Journal of Architectural and Planning Research 17, 221-235.

Shimoyama, S.: 1976, Translation of Sakuteiki: The Book of the Garden (Tokyo: Town and City Planners). Attributed to Toshitsuna Tachibana, late 11th/early 12th Centrury.

Shingen: 1466 (original text)/1930, Senzui Narabi ni yagyou no zu (Illustrations for designing mountain, water and hillside field landscapes). Manuscript dated 1466, published in Sonkeikaku Library, Sonkeikaku Soukan series. Tokyo: Ikutoku Zaidan.

Taylor A. P. Micolich and D. Jonas1999Fractal Analysis of Pollock's Drip PaintingsNature399422.

Van Tonder, G. J. and Y. Ejima: 2003. 'Flexible Computation of Shape Symmetries', IEEE Transactions on Systems, Man \& Cybernetics, Part B 33(3), 535-541.

Van Tonder, G. J. and Y. Ejima.: 2000. 'From Image Segmentation to Anti-textons', Perception 29, 1231-1247.

Van Tonder G., J. M. J. Lyons and Y. Ejima: 2002. 'Visual Structure of a Japanese Zen garden', Nature 419, 359-360.

Wertheimer, M.: 1938, 'Laws of organization in perceptual forms', in W.D. Ellis (ed.), A sourcebook of Gestalt psychology, London, UK: Routledge and Kegan Paul, pp. 71-88.

Zucker, S. W. and S. Davis: 1988. 'Points and Endpoints: A Size/Spacing Constraint for Dot Grouping', Perception 17, 229-247. 